\title{
From benign adrenal incidentaloma to adrenocortical carcinoma: an exceptional random event
}

\author{
I Belmihoub ', S Silvera², M Sibony ${ }^{3}$, B Dousset ${ }^{4}$, P Legmann5', X Bertagna1, \\ J Bertherat ${ }^{1,6}$ and G Assié ${ }^{1,6}$
}

1Department of Endocrinology, Center for Rare Adrenal Diseases, Hôpital Cochin, Assistance Publique Hôpitaux de Paris, Paris, France, ${ }^{2}$ Department of Radiology, Saint Joseph Hospital, Paris, France, Departments of ${ }^{3}$ Pathology, ${ }^{4}$ Digestive and Endocrine Surgery and ${ }^{5}$ Radiology A, Hôpital Cochin, Assistance Publique Hôpitaux de Paris, Paris, France, and ${ }^{6}$ Institut Cochin, Institut National de la Santé et de la Recherche Médicale U1016, Centre National de la Recherche Scientifique UMR8104, Université Paris Descartes, Sorbonne Paris Cité, Paris, France

\author{
Correspondence \\ should be addressed \\ to $G$ Assié \\ Email \\ guillaume.assie@aphp.fr
}

\begin{abstract}
New European guidelines for the management of adrenal incidentalomas were recently released. One of the most novel recommendations is to stop following patients when they present a typical, small and non-secreting adenoma. We report here the case of a 71-year-old man with such an adenoma, who developed an adrenocortical carcinoma (ACC) fourteen years later, with subsequent metastases and death. Clinically, he had a normal blood pressure and no sign of hormonal hypersecretion. The hormonal work-up showed no hormone excess: urinary free cortisol level was normal, the diurnal cortisol rhythm was respected and urinary catecholamine metabolites levels were normal. Computed tomography (CT) scan showed a homogeneous lesion, with a low density. The lesion remained unchanged during the five years of follow-up. Eight years after the last CT, a large right heterogeneous adrenal mass was incidentally discovered during an ultrasound examination. On CT scan, it was a $6 \mathrm{~cm}$ heterogeneous tumor. On hormonal work-up, there was no secretion. The patient was operated of an adrenalectomy, and the histology described an ACC with a Weiss score at 8, with no benign contingent. To our knowledge, this is the first case of an ACC occurring in a patient with prior adrenal imaging showing a typical benign adenoma.
\end{abstract}

\section{Introduction}

Adrenal incidentaloma is defined as an adrenal lesion, generally of $1 \mathrm{~cm}$ or more, discovered on imaging performed for extra-adrenal disorders. In medical series, $80 \%$ of adrenal incidentalomas are adenomas and $8 \%$ are carcinomas, whereas in surgical series, those rates are $55 \%$ and $11 \%$ respectively $(1,2)$. Management of adrenal incidentaloma is aimed at ruling out malignancy and hormone secretion, based on proper imaging and appropriate hormonal exploration. The European Society of Endocrinology Clinical Practice Guideline published in collaboration with the European Network for the Study of Adrenal Tumors new guidelines in 2016 for the clinical
() 2017 European Society of Endocrinology Printed in Great Britain management of patients with adrenal incidentalomas. One of the most important new recommendations is to stop the follow-up when the mass is described as a typical benign non-secreting adrenal adenoma. Such an adenoma consists in a homogeneous mass smaller than $4 \mathrm{~cm}$ with low density on CT scan and no hormone secretion (3).

We report here the case of a patient with an adrenal incidentaloma characterized as a typical benign adenoma, without any hormone secretion, who presented thirteen years later an ACC, with subsequent metastases and a fatal evolution. 


\section{Methods}

Hormones were measured as follows: ACTH was assayed by immunoradiometric assays, plasma cortisol by competition assays and urinary metanephrines were assessed by high-performance liquid chromatography. Computed tomography scans (CT scans) were performed during the five first years with unenhanced imaging to measure the largest diameter in millimeters and the basal density in Hounsfield Units (UH), and eight years later with enhanced and unenhanced acquisitions to calculate the wash out. Magnetic resonance imaging (MRI) was first performed on coronal T2-weighted imaging and fifteen years later with T2-weighted and out-of-phases images for the characterization of the adrenal lesion. MIBG scintigraphy, adrenal iodocholesterol scintigraphy and 18-fluorodeoxyglucose positron emission tomography/CT (PET/CT) were also performed. Microscopic pathological examination was realized following the criteria of the Weiss score.

\section{Case report}

A 71-year-old man was referred to the endocrinology department for the characterization of a right adrenal incidentaloma, discovered on a prior abdominal ultrasound examination in the context of urinary tract infection.

He reported a medical history of myelodysplastic syndrome with anemia and thrombocytopenia, and recurrent urinary tract infections. Clinically, the patient presented no hypertension, no clinical features of Cushing's syndrome or of catecholamine hypersecretion. Potassium level was $3.9 \mathrm{mmol} / \mathrm{L}(3.5-5 \mathrm{mmol} / \mathrm{L})$. The endocrine work-up revealed normal 24-h urine cortisol

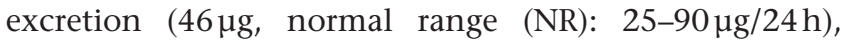
normal plasma cortisol circadian rhythm, with a midnight cortisol at $31 \mathrm{ng} / \mathrm{mL}$ and an early-morning cortisol at $130 \mathrm{ng} / \mathrm{mL}$. Both $24 \mathrm{~h}$ urinary meta- and normetanephrins, and the urinary ratio of meta- and normetanephrins to creatinine were normal. No dexamethasone suppression test was performed at this time.

The adrenal CT scan showed a right adrenal lesion measuring $17 \mathrm{~mm}$ of diameter, homogeneous. Three additional unenhanced adrenal CT scans were performed during the following five years, showing a stable tumor of $21 \mathrm{~mm}$, with low attenuations measured at 7.9, 13 and $9 \mathrm{HU}$ at 1, 3 and 5 years respectively. An MRI showed a $21 \mathrm{~mm}$ lesion, with a normal $\mathrm{T}_{2}$-weighted signal (Fig. 1), but no in and out-of-phase images were obtained at that time. The monitoring of this non-secreting adrenocortical adenoma was stopped after 5 years of follow-up, without any clinical, biological or imaging changes.

Despite the interrupted CT scan surveillance, annual ultrasound examinations were pursued in the context of recurrent urinary tract infections. Eight years after the last CT scan, a $6 \mathrm{~cm}$ heterogeneous right adrenal mass was reported.

Clinically, the patient presented no sign of hormone excess. Blood pressure was normal. Potassium level remained normal at $4.1 \mathrm{mmol} / \mathrm{L}(3.5-5 \mathrm{mmol} / \mathrm{L})$. On hormonal work-up, plasma cortisol was not suppressed

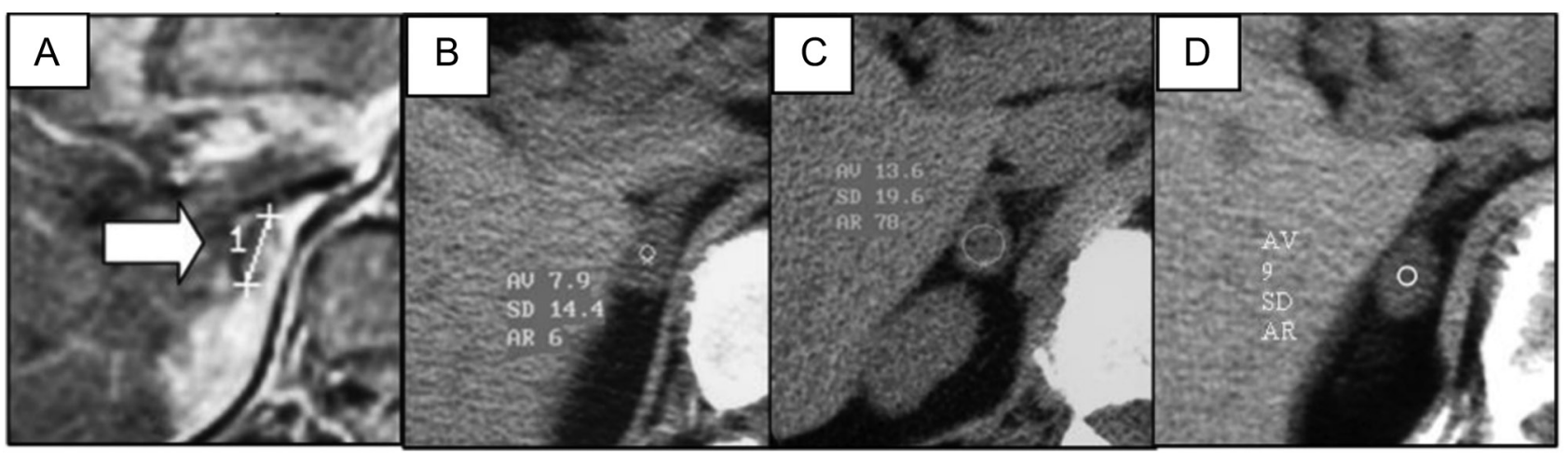

Figure 1

Adrenal lesion during the first five years of follow-up. The first image (A) is a T2-weighted image obtained during the first hospitalization, showing a well-limited right adrenal lesion (white arrow). The second image one year later is from an unenhanced CT (B) showing an adrenal lesion measured at $22 \mathrm{~mm}$, with attenuation in the region of interest of $8 \mathrm{UH}$, a finding indicative of a lipid-rich adenoma. Density values and size of the adrenal lesion remained stable three (C) and five (D) years later. 


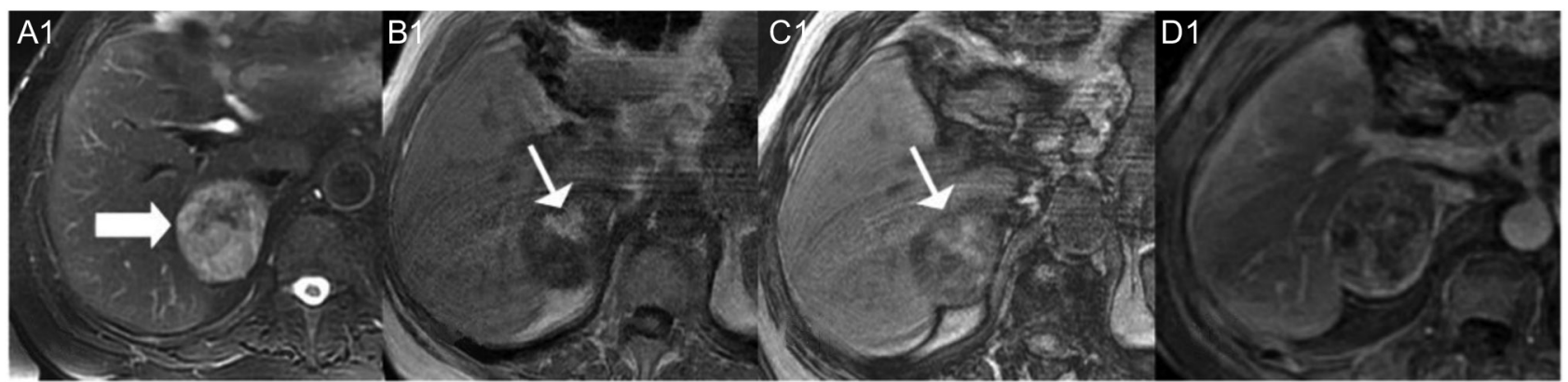

\section{Figure 2}

MR images of the adrenocortical carcinoma 15 years after the first evaluation. The right adrenal mass is hyperintense on T2-weighted sequence (A1). There is no loss of signal on out-of-phase image (C1), as compared with in-phase image (B1). The hypersignal observed in the middle of the lesion is part of a hemorrhage component (B1, C1 and white thin arrows). On late contrast enhancement images, after $5 \mathrm{~min}$, the mass appears heterogeneous and poorly enhanced (D1).

on $1 \mathrm{mg}$ overnight dexamethasone suppression test $(84 \mathrm{ng} / \mathrm{mL})$. Urinary free cortisol was normal at $46 \mu \mathrm{g} / 24 \mathrm{~h}$ (NR: 25-90 $\mu \mathrm{g} / 24 \mathrm{~h}$ ), urinary metanephrine level was $0.46 \mu \mathrm{mol} / 24 \mathrm{~h}$ (NR: $0.25-1.75 \mu \mathrm{mol} / 24 \mathrm{~h}$ ) and urinary normetanephrine level was slightly increased at $2.74 \mu \mathrm{mol} / 24 \mathrm{~h}$ (NR: $0.5-2.4 \mu \mathrm{mol} / 24 \mathrm{~h}$ ). On CT scan and MRI (Fig. 2), the adrenal mass was measuring $6 \mathrm{~cm}$. The lesion appeared heterogeneous, precluding any global density measurement. The tissular portion presented an unenhanced attenuation of $25 \mathrm{HU}$ with an absolute washout of less than $50 \%$ on CT, and insufficient signal loss on out-of-phase images compared with in-phase images on MRI.
MIBG scintigraphy and adrenal iodocholesterol scintigraphy showed no uptake on the right adrenal mass. Positron emission tomography/CT (PET/CT) images revealed a strong increased ${ }^{18}$ Fluorodeoxyglucose (FDG) uptake of the right adrenal mass, with a standardized uptake value (SUV) of 7.4. There was no other uptake.

Right adrenalectomy was performed for a suspicion of ACC. Histology confirmed the diagnosis of ACC with a Weiss score at 8 (Fig. 3). Macroscopic and microscopic examination did not identify any benign area.

Five months later, a CT scan revealed local and distal recurrence with liver, pulmonary and peritoneal metastases. The patient died 2 months later.

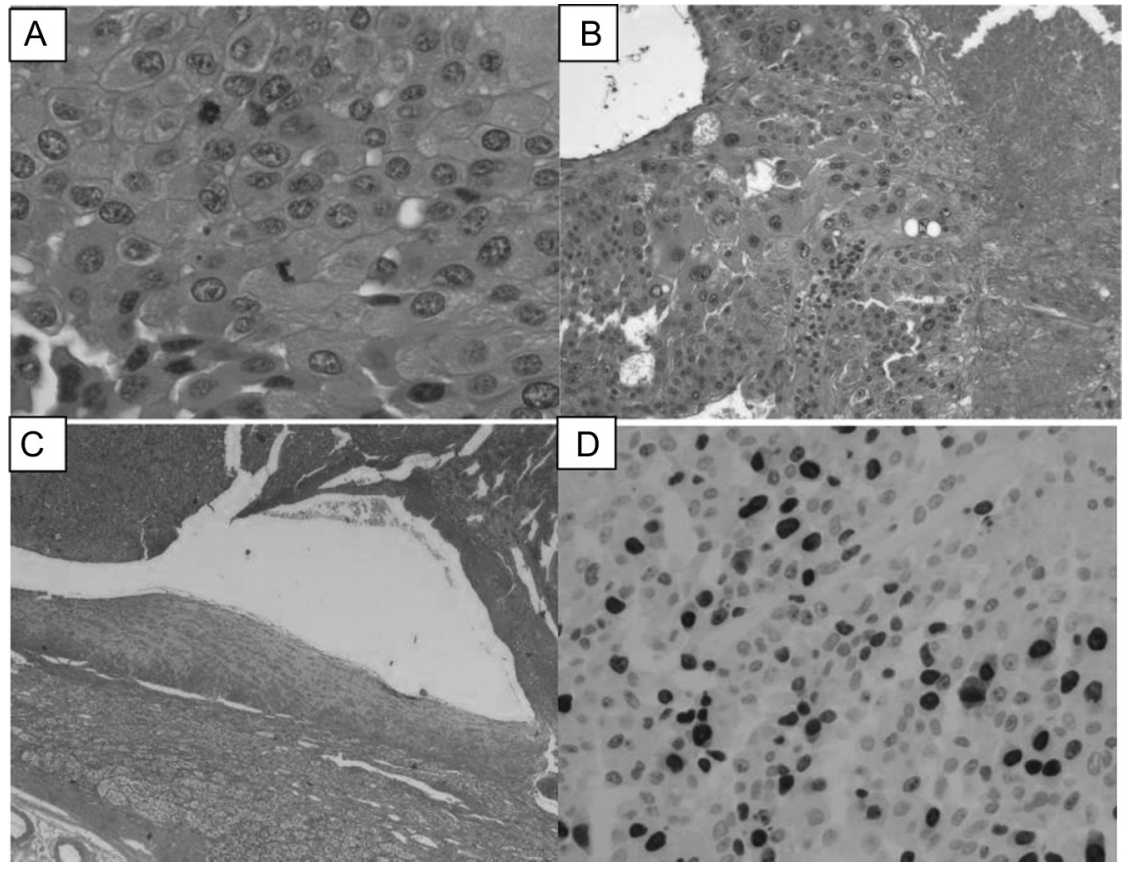

\section{Figure 3}

Histological findings after adrenal surgery. The WEISS score was 8, with 1 point for the following items: nuclear grade, high mitotic rate $(A, B)$, atypical mitotic figures, $<25 \%$ or vacuolated cells, diffuse architecture, necrosis (B), venous invasion (C), invasion of tumor capsule. There was no sinusoid invasion. Ki67-positive cells was elevated ( $30 \%$ ) (D). No benign component could be found on the adrenal. 


\section{Discussion}

In this case, the patient presented a typical benign adrenal adenoma: smaller than $4 \mathrm{~cm}$, homogeneous, with a low density on CT scan and without any hormone hypersecretion.

In its consensus statement of 2008, the French Society of Endocrinology recommended for those typical adrenal masses repeat CT imaging at 6 months, 2 and 5 years (4). The aim of this approach was to avoid missing a malignant lesion. The new European recommendations published in 2016 suggest stopping follow-up for typical benign and non-secreting adrenal adenomas. This strategy is justified, beyond the lack of malignant transformation reported so far, by the financial and psychological costs (1), and by the risk related to radiation exposure when repeated CTs are proposed (5). Despite following the 2008 recommendations, including a prolonged follow-up, we were not able to detect this ACC early. Indeed this ACC appeared later than the 5-year surveillance we performed. Of course, following the new ESE recommendations, we would also have missed this ACC.

Several reports have presented cases of ACC patients with adrenal imaging performed a year or more before the diagnosis of ACC. Oszari et al. studied 25 ACCs with previous imaging (6). Twenty of them had preexisting adrenal masses described before ACC diagnosis. However, none of these cases showed any benign radiological features on these previous images: 70\% were heterogeneous and, when performed, the pre-contrast density was $>10 \mathrm{HU}$. Nogueira et al. studied radiographic characteristics of 20 adrenal masses before ACC diagnosis (7). Only 2 of them had at least one morphological feature of benignancy, but unenhanced CT scan density was available for these 2 cases. We may speculate that spontaneous density could have helped to classify these tumors as atypical adrenal masses. In contrast, our patient had a 5-year follow-up with a well-characterized adrenal mass showing concordant features of benignancy. Therefore, a missed diagnosis of ACC at that time is unlikely.

In this case, the tumor had only a malignant component with no benign compartment, confirmed by a microscopic examination of the entire adrenal. From this observation, two hypotheses can be proposed. The first and most probable hypothesis is the independent occurrence of two events in a single adrenal, with initially a typical benign non-secreting adenoma, as demonstrated by imaging - and several years after the occurrence of an ACC - as proven histologically. In such a setting, the absence of benign compartment could be explained by an invasion and destruction of benign structures. However, instances with both an ACC and a remaining benign compartment have been reported, referred to as collision tumors (8). Bernard et al. described an adrenocortical tumor with two different histological components: one central malignant part surrounded by another part with benign tissue (9). A second hypothesis is a transformation of the adenoma into an ACC. Transformation of benign adrenal adenomas into ACC has been long debated. There are some evidence against, like the high prevalence of adenomas contrasting with the extremely low prevalence of ACC, though this does not preclude the hypothesis of exceptional transformation events or the lack of adenomatous areas in adrenals of ACC patients in most cases. On the other hand, the adenoma-carcinoma sequence, similar to the pathogenesis of colorectal cancer, is supported by some molecular evidence. First, many adenomas and ACC share common molecular alterations, such as beta-catenin mutations $(10,11)$. Furthermore, using mouse models, two studies showed that activating beta-catenin and Igf2 led to a sequence of benign adrenal proliferation, evolving into malignant proliferation at later stages $(12,13)$. Finally in human tumors, Ronchi et al. investigated 46 adrenal tumors - adenomas and carcinomas - and reported genes pathways, such as Wnt/beta-catenin and also Notch signaling similarly altered in ACA and ACC, compatible with an adenomacarcinoma sequence (14). Altogether, these observations suggest that ACC may develop from adenomas, but this process is obviously rare and further studies are needed for a definite proof of the adenoma-carcinoma sequence in the adrenal cortex.

To conclude, the case reported here of an ACC occurring several years after the diagnosis of a typical adrenal adenoma as highly exceptional. Should this single case alleviate the new ESE consensus defending the total absence of surveillance for typical adrenal adenomas? We do think that these new guidelines should not be modified based on a single case, especially considering the more than two thousands adenomas with no malignant transformation reported in literature.

\footnotetext{
Declaration of interest

The authors declare that there is no conflict of interest that could be perceived as prejudicing the impartiality of this case report.
}

Funding

The authors thank Brou de Lauriere foundation, COMETE-TACTIC (grant PRT-K 14079, grant INCA_DGOS 8663), and Carigest-promex for their support. 


\section{Consent statement}

We declare that consent has not been obtained because the patient is deceased.

\section{References}

1 Barzon I, Sonino N, Fallo F, Palu G \& Boscaro M. Prevalence and natural history of adrenal incidentalomas. European Journal of Endocrinology 2003149 273-285. (doi:10.1530/eje.0.1490273)

2 Kloos RT, Gross MD, Francis IR, Korobkin M \& Shapiro B. Incidentally discovered adrenal masses. Endocrine Reviews 199516 460-484. (doi:10.1210/edrv-16-4-460)

3 Fassnacht M, Wiebke A, Bancos I, Dralle H, Newell-Price J, Sahdev A, Tabarin A, Terzolo M, Tsagarakis S \& Dkkers OM. Management of adrenal incidentalomas: European Society of Endocrinology Clinical Practice Guideline in collaboration with the European Network for the Study of Adrenal Tumors. European Journal of Endocrinology 2016 175 G1-G34. (doi:10.1530/EJE-16-0467)

4 Tabarin A, Bardet S, Bertherat J, Dupas B, Chabre O, Hamoir E, Laurent F, Tenenbaum F, Cazalda M, Lefebvre $\mathrm{H}$ et al. Exploration and management of adrenal incidentalomas, French Society of Endocrinology Consensus. Annales d'Endocrinologie 200869 487-500. (doi:10.1016/j.ando.2008.09.003)

5 Cawood Tj, Hunt PJ, O'Shea D, Cole D \& Soule S. Recommended evaluation of adrenal incidentalomas is costly, has high positive rates and confers a risk of a fatal cancer that is similar to the risk of the adrenal lesion becoming malignant: time for a rethink? European Journal of Endocrinology and Metabolism 201196 2004-2016. (doi:10.1210/jc.2011-0085)

6 Oszari L, Kutahyalioglu M, Elsayes KM, Vicens RA, Sircar K, Jazaerly T, Waguespack SG, Busaidy NL, Cabanillas ME, Dadu R et al. Preexisting adrenal masses in patients with adrenocortical carcinoma: clinical and radiological factors contributing to delayed diagnosis. Endocrine 201651 351-359. (doi:10.1007/s12020-015-0694-7)
7 Nogueira TM, Lirov R, Caoili EM, Lerario AM, Miller BS, Fragoso MC, Dunnick NR, Hammer GD \& Else T. Radiographic characteristics of adrenal masses preceding the diagnosis of adrenocortical cancer. Hormones and Cancer 20156 176-181. (doi:10.1007/s12672-015-0225-2)

8 Katabathina VS, Flaherty E, Kaza R, Ojili V, Chintapalli KN $\&$ Prasad SR. Adrenal collision tumors and their mimics: multimodality imaging findings. Cancer Imaging 201313 602-610. (doi:10.1102/1470-7330.2013.0053)

9 Bernard MH, Sidhu S, Berger N, Peix JL, Marsh DJ, Robinson BG, Gaston V, Le Bouc Y \& Gicquel C. A case report in favor of a multistep adrenocortical tumorigenesis. Journal of Clinical Endocrinology and Metabolism 200388 998-1001. (doi.org/10.1210/jc.2002-021117)

10 Ronchi CL, Di Dalmazi G, Faillot S, Sbiera S, Assié G, Weigand I, Calebiro D, Schwarzmayr T, Appenzeller S, Rubin B et al. Genetic landscape of sporadic unilateral adrenocortical adenomas without PRKACA p.Leu206Arg mutation. Journal of Clinical Endocrinology and Metabolism 2016 101 3526-3538. (doi:10.1210/jc.2016-1586)

11 Assié G, Letouzé E, Fassnacht M, Jouinot A, Luscap W, Barreau O, Omeiri H, Rodriguez S, Perlemoine K, René-Corail F et al. Integrated genomic characterization of adrenocortical carcinoma. Nature Genetics 201446 607-612. (doi:10.1038/ng.2953)

12 Heaton JH, Wood MA, Kim AC, Lima LO, Barlaskar FM, Almeida MQ, Fragoso MC, Kuick R, Lerario AM, Simon DP et al. Progression to adrenocortical tumorigenesis in mice and humans through insulinlike growth factor 2 and $\beta$-catenin. American Journal of Pathology 2012 181 1017-1033. (doi:10.1016/j.ajpath.2012.05.026)

13 Drelon C, Berthon A, Ragazzon B, Tissier F, Bandiera R, SahutBarnola I, de Joussineau C, Batisse-Lignier M, Lefrançois-Martinez $\mathrm{AM}$, Bertherat J et al. Analysis of the role of Igf2 in adrenal tumour development in transgenic mouse models. PLOS ONE 20127 e44171. (doi:10.1371/journal.pone.0044171)

14 Ronchi CL, Sbiera S, Leich E, Henzel K, Rosenwald A, Allolio B \& Fassnacht M. Single nucleotide polymorphism array profiling of adrenocortical tumors - evidence for an adenoma carcinoma sequence? PLoS ONE 20138 e73959. (doi:10.1371/journal. pone.0073959)

Received 15 January 2017

Revised version received 9 March 2017

Accepted 27 March 2017 